Rabaska

Revue d'ethnologie de l'Amérique française

Castonguay, Jacques. La Saga de la navigation à Québec et sur le Saint-Laurent. Sillery, Éditions du Septentrion, 2007, 118 p. incluant un glossaire. ISBN 2-89448-526-3

\title{
André Kirouac
}

Volume 6, 2008

URI : https://id.erudit.org/iderudit/000021ar

DOI : https://doi.org/10.7202/000021ar

Aller au sommaire du numéro

Éditeur(s)

Société québécoise d'ethnologie

ISSN

1703-7433 (imprimé)

1916-7350 (numérique)

Découvrir la revue

Citer ce compte rendu

Kirouac, A. (2008). Compte rendu de [Castonguay, Jacques. La Saga de la navigation à Québec et sur le Saint-Laurent. Sillery, Éditions du Septentrion, 2007, 118 p. incluant un glossaire. ISBN 2-89448-526-3]. Rabaska, 6, 164-166. https://doi.org/10.7202/000021ar d'utilisation que vous pouvez consulter en ligne.

https://apropos.erudit.org/fr/usagers/politique-dutilisation/ 
Outre le texte des auteurs qui est d'une grande qualité, cette publication comprend de nombreux extraits de publications anciennes comme les Relations des jésuites, ou encore des textes de Louis Fréchette et de l'abbé Honorius Provost, lesquels évoquent les Noëls anciens. On y reproduit aussi des articles publiés dans des journaux et des magazines de diverses époques, comme Le Monde illustré, La Revue populaire et Le Samedi. Les auteurs ont aussi intégré à leur ouvrage de nombreux témoignages contemporains qui contribuent à faire le pont entre le passé et le présent. Mentionnons celui de Marie Guérard intitulé « Toc, toc, toc : le Père Noël arrive », dans lequel elle raconte la visite du Père Noël chez elle à la fin des années 1950, ou encore celui de Denis Robitaille, «Un Noël réinventé », qui nous rappelle que les Noëls d'aujourd'hui ressemblent de moins en moins à l'image que l'on a du Noël québécois traditionnel.

Dans leur exploration de la fête de Noël au Québec, Sylvie Blais et Pierre Lahoud arrivent à la constatation que Noël est vraiment la grande fête de la nostalgie. « Nous mettons un peu des Noëls passés dans chaque Noël d'aujourd'hui. » Ils soulignent aussi que chaque génération a tendance à se plaindre de l'abandon des bonnes vieilles traditions : « Les Noëls d'antan sont toujours plus extraordinaires qu'ils datent de 1850 ou de 1970 ! » Mais ce qui ressort le plus dans leur recherche, ce sont les origines diversifiées des traditions de Noël au Québec : «Au rythme des mutations, des inventions et des abandons, Noël constitue, plus que tout autre fête, un alliage dont les apports culturels sont multiples. »

La Fête de Noël au Québec est un très beau livre basé sur une solide recherche menée avec passion. Il demeurera pendant longtemps le livre de référence sur cette fête dans la Belle Province. À souhaiter qu'une publication semblable sur le Premier de l'an verra bientôt le jour.

Georges Arsenault

Charlottetown

Castonguay, JAcques. La Saga de la navigation à Québec et sur le SaintLaurent. Sillery, Éditions du Septentrion, 2007, 118 p. incluant un glossaire. ISBN 2-89448-526-3.

Régis Boyer (L'Islande médiévale, p. 189) a donné la définition suivante de la saga : «On appelle saga un récit en prose, toujours en prose, ce point est capital, rapportant la vie et les faits et gestes d'un personnage, digne de mémoire pour diverses raisons, depuis sa naissance jusqu'à sa mort, en n'omettant ni ses ancêtres ni ses descendants s'ils ont quelque importance ». 
Notons encore qu'une saga n'est qu'extrêmement rarement une légende ou un conte, d'après l'encyclopédie Wikipédia.

Peut-on dire du volume de Jacques Castonguay La Saga de la navigation à Québec et sur le Saint-Laurent qu'il s'agit du récit d'une saga ? Par extension, nous comprendrons que le personnage central est le Saint-Laurent. Par contre, en 118 pages, peut-on espérer que rien ne sera omis ? Le désir de l'auteur semble ambitieux et force est d'admettre que ce livre n'a rien du récit d'une saga!

Pourrait-on y voir un lien avec l'emphase que l'auteur met sur la question de la présence des Vikings car l'un des premiers chapitres s'intitule «Les drakkars des Vikings également dans le Saint-Laurent»? À moins que Jacques Castonguay ne connaisse des sources non encore décrites, aucune preuve de la présence des Vikings n'est attestée dans le Saint-Laurent, sinon que sur la rive atlantique de Terre-Neuve. Encore là, il faut définir ce que l'on entend par l'étendue du grand fleuve. Pour les fins de cette critique, nous entendrons par Saint-Laurent toute la portion allant des Grands Lacs jusqu'aux limites du golfe, car l'auteur ne semble pas parler du fleuve spécifiquement.

Autant une grande place est réservée aux Vikings, autant peu est dit sur les Basques alors que de nombreuses preuves démontrent qu'ils ont remonté le fleuve au moins jusqu'à Trois-Pistoles. Une «saga » ne peut omettre la présence basque quand l'auteur souligne l'importance des Phéniciens. Ce dernier peuple, quoique grand navigateur, est incongru dans un volume ayant ce thème.

L'ambition de l'écriture d'une saga permet de penser, au moins, que l'on y verra des mentions importantes de grands pans de l'histoire du fleuve. Quelques lignes sur le transport par goélettes, autant à voile qu'à moteur ; rien concernant la voie maritime ou la construction navale. L'accent est mis sur la Première Guerre mondiale alors que la présence des U-Boote allemands pendant la Seconde est l'un des grands moments de l'histoire du fleuve. D'ailleurs, ces sous-marins furent présents en eaux canadiennes en 1942 et 1944, et non en 1943 comme l'auteur l'écrit.

De grands pans d'histoire sont manquants et remplacés plutôt par diverses anecdotes dont plusieurs touchent le thème des croisières. Il semble de plus que l'auteur, dans ce dernier cas, a puisé dans ses collections personnelles d'archives et a trop longuement élaboré sur la thématique si on en compare l'ampleur avec les autres chapitres.

Au niveau de la rigueur des informations écrites, il faut s'en remettre à Jacques Castonguay, car peu de sources, étayant les points historiques, sont présentées. Les photographies, à la résolution douteuse, comportent toutefois des indications de provenance, bien qu'il faille par exemple déduire que $\mathrm{CP}$ 
doit désigner le Canadien Pacifique.

Dans l'ensemble, le volume de Jacques Castonguay laisse le lecteur sur un appétit inassouvi et un doute sur bon nombre d'affirmations.

André Kirouac

Musée naval de Québec

Champagne, Juliette-Marthe. De la Bretagne aux plaines de l'Ouest canadien. Lettres d'un défricheur franco-albertain, Alexandre Mahé (1880-1968). Québec, Presses de l'Université Laval, «Intercultures », 2003, 317 p. ISBN 2-7637-7924-7.

Issue d'une thèse de doctorat soutenue à l'Université Laval en 2001, cette biographie d'un Breton immigré dans le nord-est de l'Alberta en 1909 est riche de renseignements sur la vie quotidienne et l'univers mental des colons de langue française établis dans les provinces des Prairies. Empruntant sa méthodologie à la micro-histoire, Juliette Champagne montre qu'il est possible, à partir de documents épars, de reconstruire l'expérience des gens humbles, à condition de savoir faire parler les sources. Sa double formation d'historienne et d'ethnologue lui rend de grands services.

Champagne a organisé sa matière en dix chapitres. Dans un premier temps, elle aborde des questions méthodologiques, notamment le fait d'étudier nul autre que son grand-père maternel ; ce chapitre lui donne l'occasion de traiter de l'historiographie des Canadiens français de l'Ouest et de présenter sa documentation. Champagne consacre son deuxième chapitre aux origines et à la formation d'Alexandre Mahé dans le «pays de mémoire » qu'est la Bretagne, insistant particulièrement sur son engagement de catholique et sur son séjour en Afrique. Ensuite, l'auteur amène le lecteur au côté d'Alexandre Mahé lors de son voyage successivement transatlantique et transcontinental, puis son installation dans la région de Saint-Paul-des-Métis. La colonisation de ce terrritoire constitue la matière du quatrième chapitre de De la Bretagne aux plaines de l'Ouest canadien, particulièrement les activités agricoles et commerciales de Mahé, qui allie, pendant presque dix ans, les métiers de cultivateur et de marchand général. Au chapitre suivant, Champagne approfondit le sujet du « cultivateur et sa ferme » (règlement des dettes au magasin, agrandissement de la ferme et sa mise en valeur, employés, viabilité de la ferme). Le chapitre six porte sur la paroisse et la communauté de SaintVincent, et le chapitre sept sur le rôle social de Mahé ; celui-ci participe en effet à la fondation et à l'essor de l'Association canadienne-française de l'Alberta (ACA) et il s'intéresse de près aux progrès du journal La Survivance, 